Recibido: 08/05/2019 --- Aceptado: 27/03/2020 --- Publicado: 15/07/2020

\title{
IMPORTANCIA DE LOS ESTUDIOS DE OPINIÓN PÚBLICA: SU APLICACIÓN Y SEGMENTACIÓN DEL MERCADO EN MÉXICO ${ }^{1}$
}

\section{IMPORTANCE OF PUBLIC OPINION STUDIES: THEIR APPLICATION AND SEGMENTATION OF THE MARKET IN MEXICO}

\begin{abstract}
Alejandra Zarzosa Codocedo². Centro Universitario de Ciencias Económico Administrativas. México. Alejandra.Zarzosa@redudg.udg.mx
\end{abstract}

Rosario Cota-Yáñez. Centro Universitario de Ciencias Económico Administrativas. México. maria.cota@academicos.udg.mx

Ulises Rodríguez Cota. Pasante de la licenciatura en Comunicación y Periodismo Deportivo del Instituto José Ramón Fernández. México.

ulises.101@hotmail.com

\section{RESUMEN}

El objetivo del presente trabajo comprende la realización de un análisis descriptivo de la industria de los estudios de opinión pública y de mercado en México, las principales características del mercado desde la perspectiva de la oferta y la demanda, así como la segmentación del mercado por tipo de producto. La metodología implementada consiste en la aplicación de entrevistas a profundidad enfocadas a diversos sectores, como son: servidores públicos, candidatos en comicios electorales, asesores y estrategas de campaña, expertos académicos, directores de áreas de gestión de información e investigación en el sector privado, tales como directores de agencias y consultores independientes en materia de investigación de mercados y opinión pública. El resultado final trata de ofrecer una visión acerca de la dimensión, especialización y profesionalización de esta industria en constante auge en México. Se presentan a los estudios de opinión pública y de mercado como una herramienta indispensable para la recolección de información, análisis y toma de decisiones, que permiten medir las preferencias de los

\footnotetext{
${ }^{1}$ Este trabajo resume parte de los resultados del estudio de caso: "Análisis del mercado local de los estudios de opinión pública y mercados, y factores que inciden en su credibilidad", presentado en la Maestría en Mercadotecnia Global del Instituto Tecnológico y de Estudios Superiores de Occidente.

2 Alejandra Zarzosa Codocedo: Profesora Investigadora de la Universidad de Guadalajara, Maestra en Mercadotecnia Global por el Instituto Tecnológico y de Estudios Superiores de Occidente. Licenciada en Mercadotecnia.

Alejandra.Zarzosa@redudg.udg.mx
} 
demandantes en cualquier ámbito. Del mismo modo que muestra, explica y analiza cuál es la relación y el grado de importancia que emanan de ellos; su importancia radica que en los últimos años, han cobrado vigencia en diversos segmentos del mercado, como lo son el electoral, el público y el privado, entre otros.

PALABRAS CLAVE: estudio de opinión - preferencias electorales - segmentación de mercado - mercadotecnia electoral - encuestas de salida - oferta - demanda.

\section{ABSTRACT}

The objective of this work includes the carrying out of a descriptive analysis of the industry of public opinion and market studies in Mexico, the main characteristics of the market from the perspective of supply and demand, as well as market segmentation by type of product. The implemented methodology consists of the application of in-depth interviews focused on various sectors, such as public servants, candidates in electoral elections, campaign advisers and strategists, academic experts, directors of areas of information management and research in the private sector, such as agency directors and independent consultants on market research and public opinion. The final result tries to offer a vision about the dimension, specialization and professionalization of this industry in constant growth in Mexico. They are presented to public opinion and market studies as an indispensable tool for information gathering, analysis and decision-making, which make it possible to measure the preferences of the plaintiffs in any field. In the same way that it shows, explains and analyzes what is the relationship and the degree of importance that emanate from them; Its importance lies in the fact that in recent years, they have come into force in various market segments, such as the electoral, public and private sectors, among others.

KEYWORDS: opinion study - electoral preferences - market segmentation electoral marketing - exit surveys - offer - demand.

\section{IMPORTÂNCIA DOS ESTUDOS DE OPINIÃO PÚBLICA: A SUA APLICAÇÃO E SEGMENTAÇÃO DO MERCADO NO MÉXICO}

\section{RESUMO}

O objetivo do presente trabalho inclui a realização de uma análise descritiva da indústria dos estudos de opinião pública e de mercado no México, as principais características do mercado desde a perspetiva da oferta e a demanda, assim como a segmentação do mercado por tipo de produto. A metodologia implementada consiste na aplicação de entrevistas a profundidade focadas aos vários setores, sendo eles: servidores públicos, candidatos em eleições, assessores e estrategistas de campanha, especialistas acadêmicos, diretores de áreas de gestão de informação e pesquisa no setor privado, tais como diretores de agências e consultores independentes em matéria de pesquisa de mercados e opinião pública. $O$ resultado final tenta fornecer uma visão sobre a dimensão, especialização e profissionalização desta indústria em constante auge no México. Se apresentam os estudos de opinião 
pública e de mercado como uma ferramenta indispensável para a coleta de informação, análise e tomada de decisões, que permitem mensurar as preferências dos requerentes em qualquer âmbito. Da mesma forma que mostra, explica e analisa qual é a relação e o grau de importância deles; a sua importância se fundamenta em que nos últimos anos, entraram em vigor diversos segmentos do mercado, como o eleitoral, o público e o privado, entre outros.

PALAVRAS CHAVE: estudo de opinião - preferências eleitorais - segmentação de mercado - marketing eleitoral - sondagens - oferta - demanda.

\section{Cómo citar el artículo:}

Zarzosa Codocedo, A., Cota Yáñez, R. y Rodríguez Cota, U. (2020). Importancia de los estudios de opinión pública: su aplicación y segmentación del mercado en México. [Importance of public opinion studies: their application and segmentation of the market in Mexico]. Revista de Comunicación de la SEECI, (52), 95-117. doi: http://doi.org/10.15198/seeci.2020.52.95-117

Recuperado de http://www.seeci.net/revista/index.php/seeci/article/view/545

\section{INTRODUCCIÓN}

Los estudios de opinión son un instrumento útil para recabar información en el ámbito de la investigación; permiten medir y dimensionar un fenómeno. Mediante la creación de variables medibles; conocer las razones por las cuales los demandantes prefieren un producto o preferencias del voto, las necesidades de salud en el momento de sacar al mercado un nuevo producto ergonómico. Destacan dentro de los estudios de tipo pre-electoral, la encuesta de salida y el conteo rápido, así como los estudios de opinión pública. Las encuestas representan un aporte en la realización de estudios de mercado, que posteriormente servirán de insumos a otras metodologías como son: grupos focales, entrevistas de profundidad, entre otros.

Los trabajos que abordan la temática son de D'Adamo (2007); Ruíz (1997); Young (1995) y Rivadeira (1995). Quienes manifiestan un consenso con respeto al término de opinión pública y argumentan que no es nuevo, ya era utilizado desde el siglo XVIII, incluso puede ser rastreado en los trabajos de Platón y Aristóteles.

La opinión pública es un instrumento que presenta el sentir de la sociedad influenciada algunas veces por el efecto de los medios de comunicación. De acuerdo a Ruiz (1997) tiene dos formas de entendérsele: la tradicional que se enfoca por la definición e influencias de la opinión. Por ejemplo: las políticas públicas se ajusten a las demandas de la opinión pública; a diferencia la tradición empírica se enfoca a los datos que aporta un grupo representativo de personas, estudios de tendencias, inclinación política, decisiones del voto, entre otros.

La importancia de la opinión pública radica en el hecho de que condiciona el comportamiento. Young (1995, p. 11) considera que la opinión pública es una 
creencia arraigada pero menos consistente que un conocimiento tiene un carácter verbal y simbólico.

Por otra parte, la investigación cuantitativa ofrece a la investigación social, la posibilidad de conocer la opinión de la población y en particular aquella que proviene de los ciudadanos, respecto a variables que forman parte de su vida; por ejemplo la evaluación de servicios públicos, vivienda, salud, trabajo, cultura, transporte, problemas sociales. Información que representa un insumo útil para el diseño de políticas públicas y acciones de gobierno encaminadas a implementar mejoras y cambios en beneficio de la población.

En el sector privado permite realizar mediciones de mercado a quienes conforman este sector, contar con elementos que coadyuven a su competitividad y permanencia. Entre la gran diversidad de estudios de mercado que se llevan a cabo mediante la aplicación de estas herramientas destacan: estimación del mercado potencial, estimaciones de ventas, medición de la participación del mercado, conocimiento de imagen y posicionamiento, recordación de marca -top of mind-, entre otros. Las posibilidades de uso que ofrece esta herramienta son múltiples dependiendo de las necesidades en cada situación.

El Estudio Anual de la Industria de la Investigación de Mercados y Estudios de Opinión Pública en México, realizado por la AMAI en 2014 menciona que la proporción de estudios cuantitativos realizados es del $73 \%$ frente al $25 \%$ de estudios cualitativos. Fueron requeridos en el $88 \%$ de los casos por la iniciativa privada y el $12 \%$ restante por el sector público (AMAI, 2014).

Dentro de las empresas más importantes de acuerdo al ranking nacional de la Asociación Mexicana de Agencias de Investigación de Mercados y Opinión Pública, en materia de realización de proyectos de tipo cuantitativo figuran: IPSOS México, Millward Brown México, De la Riva, Estadística Aplicada e Investigación de Mercados, Brand investigation, Marketing Group, Grupo GDV, Pearson, Consulta, Berúmen y Asociados.

De acuerdo a los portales electrónicos de cada una de las empresas se encuentra que se especializan en cuatro categorías: Estudios de opinión pública, Estudios electorales, Investigación de mercado y Estrategias de mercadotecnia.

Estudios de opinión: Recolección de información de la opinión pública a través de encuestas cuyas temáticas radican principalmente acerca de: temas sociales, evaluación de gobierno y/o políticas públicas desde la perspectiva de la población, cuya técnica de investigación se fundamenta exclusivamente en las encuestas como instrumento de recolección, independientemente del análisis estadístico que se realice a posteriori.

Estudios electorales: Medición de tendencias en las preferencias electorales previas a la elección, encuesta de salida y conteo rápido. 
Investigación de Mercados: Diseño y ejecución de diferentes técnicas de investigación para la obtención de información de diferentes casos en particular. Este tipo de estudios requiere de las diferentes técnicas que en su conjunto ofrecen información con la finalidad de cubrir un objetivo particular a usuario o cliente en un momento específico.

Estrategias de Mercadotecnia: En esta categoría se considera a empresas que ofrecen una gran diversidad de productos entre las que se encuentran los estudios de carácter electoral, así como también tienen la capacidad y la experiencia para la realización de estudios de mercado, cuentan con la infraestructura para la realización de producción audio visual, diseño de campañas de publicidad, o se especializan en la medición de audiencias y/o de consumo.

Aun considerando las diferentes orientaciones no son excluyentes entre sí, con excepción de la empresa IBOPE AGB México que se enfoca exclusivamente a la medición de audiencias. Así también sucede con el caso de consulta Mitofsky que realiza investigaciones de mercado, pero tiene un importante posicionamiento en el ámbito de los estudios electorales dada su presencia en los medios de comunicación. En contraste la empresa Berúmen y Asociados, tiene un importante posicionamiento en el sector privado. Por otra parte KP Alasraki, tiene una mínima participación en los estudios electorales, por lo menos en aquellos de carácter público, así como en los estudios de opinión, sin embargo su presencia es muy fuerte en cuanto a investigación de mercados se refiere y además la diversificación de su producto se extiende hasta el diseño de campañas de publicidad, producción de medios. Investigaciones sociales aplicadas (ISA), se enfoca a la realización de estudios de opinión principalmente y a los estudios de carácter electoral pero en menor medida.

\section{OBJETIVOS DE INVESTIGACIÓN}

- Identificar la fuente de inicio de realización de búsqueda de información a través de la realización de un estudio de opinión y/o de mercado.

- Describir el proceso que lleva a cabo la organización para la realización y/o compra de un estudio de mercado.
$\checkmark \quad$ Momentos de compra.
Roles de compra: tomadores de decisiones.
Selección de proveedor/método de realización.
Elementos de comparación y decisión.

- Conocer el uso de los estudios de opinión y de mercado como herramienta en la toma de decisiones.

\section{METODOLOGÍA}

Se realizó un estudio cualitativo mediante la aplicación de entrevistas a profundidad a quienes conforman la demanda del mercado de los estudios de opinión pública y estudios de mercado; se presenta un análisis descriptivo al respecto, en particular se describe el proceso de compra identificando los diferentes 
Zarzosa Codocedo, A., Cota-Yáñez, R. y Rodríguez Cota, U. Importancia de los estudios de opinión pública: su aplicación y segmentación del mercado en México

usos y aplicaciones de esta herramienta desde la perspectiva de los diferentes segmentos que conforman el mercado (ver figura 1 ).

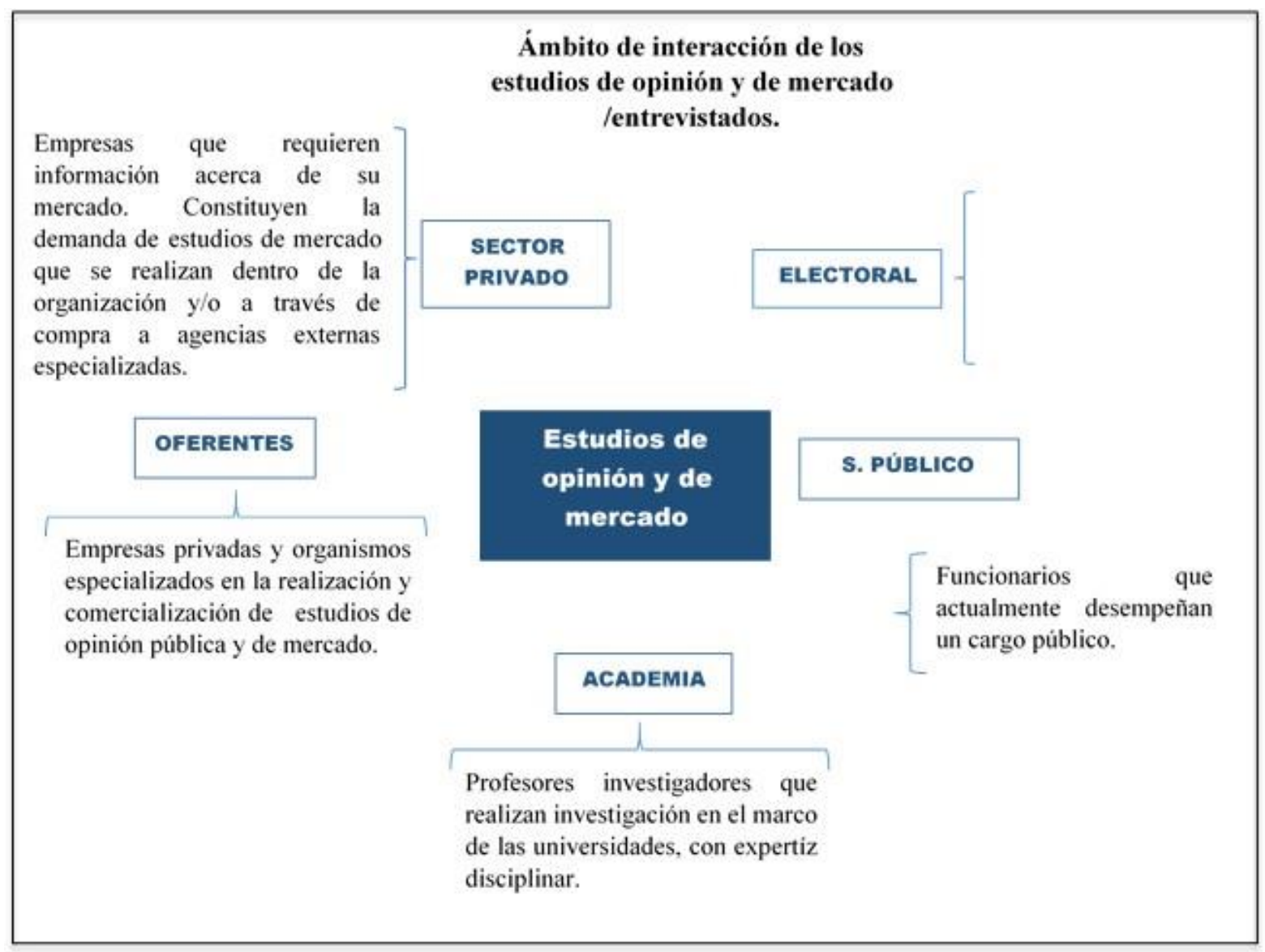

Figura 1: ámbito de interacción de los estudios de opinión y de mercado.

Fuente: elaboración propia con base a la metodología de la investigación.

Segmento 1. Electoral: profesionistas que se desempeñan en el ámbito de la política en el sector público, quienes cuentan con la experiencia de haber participado como candidatos en una contienda electoral o bien, como colaboradores en procesos de elección popular.

Segmento 2. Sector Público: Funcionarios públicos, quienes se desempeñan un cargo al servicio público.

Segmento 3. Sector Privado: Profesionistas, quienes se desempeñan en la iniciativa privada en el área propia y/o vinculada con la investigación de mercados.

Segmento 4. Académico: Profesores investigadores que desarrollan investigación en el marco de las universidades.

Segmento 5. Oferentes: Empresas privadas y organismos públicos especializados en la realización estudios de opinión pública y de mercado y en su caso la comercialización de éstos. 


\subsection{Técnica de investigación}

La técnica cualitativa de investigación en este caso permite acceder a conocer la perspectiva de la persona entrevistada detalladamente, en particular las entrevistas de profundidad semiestructuradas, se realizan cara a cara con el entrevistado y se ciñen a una estructura temática que permite guiar la temática de la entrevista asegurando la cobertura temática que responde a los objetivos (Aker, 2010, p. 189). Las entrevistas fueron audio grabadas y transcritas posteriormente para su análisis, llevaron en promedio de 20 a 30 minutos.

\subsection{Instrumento de recolección}

El instrumento de recolección comprende la guía de tópicos que plantea a manera de preguntas abiertas las preguntas de investigación entre las que destacan: ¿Quiénes conforman la oferta y la demanda de estudios de opinión y de mercado?, ¿Qué tipo de estudios requiere la demanda?, ¿Qué uso le da la demanda a los estudios de opinión y de mercado?

\subsection{Objetivos de investigación}

- Identificar la fuente de inicio de realización de búsqueda de información a través de la realización de un estudio de opinión y/o de mercado.

- Describir el proceso que lleva a cabo la organización para la realización y/o compra de un estudio de mercado.
$\checkmark \quad$ Momentos de compra.
Roles de compra: tomadores de decisiones.
Selección de proveedor/método de realización.
Elementos de comparación y decisión.

- Conocer el uso de los estudios de opinión y de mercado como herramienta en la toma de decisiones.

\subsection{Trabajo de campo}

En lo que respecta a la selección de los sujetos de estudio, un elemento de consideración y que representa un denominador común para determinar el perfil de los entrevistados comprende el hecho de que en el ámbito profesional han tomado en más de alguna ocasión, y/o actualmente toman decisiones con base en estudios de opinión y/o de mercado, o bien, se dedican profesionalmente al desarrollo de este tipo de proyectos.

Como parte del grupo integrado por los entrevistados quienes conforman el universo de estudio, se encuentran algunos casos de quienes con anterioridad se han desempeñado profesionalmente en la realización de este tipo de estudios y que, en la actualidad desempeñan funciones vinculadas al ámbito de la investigación.

Se llevaron a cabo 18 entrevistas de profundidad semiestructuradas, distribuidas entre los diferentes segmentos del mercado: 
Zarzosa Codocedo, A., Cota-Yáñez, R. y Rodríguez Cota, U. Importancia de los estudios de opinión pública: su aplicación y segmentación del mercado en México

- Segmento Electoral: 6 entrevistas.

- Segmento Sector Público: 2 entrevistas.

- Segmentos Sector Privado: 2 entrevistas.

- Segmentos Académico: 5 entrevistas.

- Segmento Oferentes: 3 entrevistas.

El siguiente cuadro describe los perfiles de los entrevistados para la presente investigación por cada segmento. Cabe hacer mención que las particularidades de algunos entrevistados se reservan a confidencialidad, a petición de algunos de ellos.

Tabla 1. Listado de entrevistas.

\begin{tabular}{|c|c|c|}
\hline $\begin{array}{l}\text { SEGMENTO } \\
\text { DEL } \\
\text { MERCADO }\end{array}$ & ENTREVISTAS & PERFIL ENTREVISTADOS \\
\hline \multirow[t]{6}{*}{$\begin{array}{l}\text { Segmento } \\
\text { Electoral }\end{array}$} & \multirow[t]{6}{*}{6 entrevistas } & $\begin{array}{l}\text { 1. Diputado Federal electo y ex coordinador de Campaña para } \\
\text { Presidente Municipal en el proceso electoral } 2015 .\end{array}$ \\
\hline & & 2. Diputado Local en funciones \\
\hline & & $\begin{array}{l}\text { 3. Secretario de Partido, ex regidor y colaborador en la campaña } \\
\text { electoral en el proceso electoral } 2015 \text {. }\end{array}$ \\
\hline & & $\begin{array}{l}\text { 4. Regidor en funciones, ex colaborador de Campaña Presidente } \\
\text { Municipal en el proceso electoral } 2015 .\end{array}$ \\
\hline & & $\begin{array}{l}\text { 5. Candidato a Presidente Municipal en el proceso electoral } \\
2015 .\end{array}$ \\
\hline & & $\begin{array}{l}\text { 6. Asesor político de Gobierno Municipal, Asesor en } \\
\text { Comunicación Estratégica, y ex Director de Estudios Políticos } \\
\text { del Centro de Opinión de la Universidad de Guadalajara. }\end{array}$ \\
\hline $\begin{array}{l}\text { Segmento } \\
\text { Público }\end{array}$ & 2 entrevistas & $\begin{array}{l}\text { 7. Director General Sistema de Tren Eléctrico Urbano SITEUR. } \\
\text { 8. Director de la Escuela Normal Superior de Jalisco. }\end{array}$ \\
\hline \multirow[t]{2}{*}{$\begin{array}{l}\text { Segmento } \\
\text { Sector } \\
\text { Privado }\end{array}$} & \multirow[t]{2}{*}{2 entrevistas } & $\begin{array}{l}\text { 9. Directora de Mercadotecnia en empresa líder en la industria } \\
\text { farmacéutica y ex Coordinadora de Planeación y Análisis } \\
\text { Estratégico de empresa líder en la industria farmacéutica. }\end{array}$ \\
\hline & & $\begin{array}{l}\text { 10. Jefe de investigación de Mercado de Corporativo líder en el } \\
\text { ramo de las telecomunicaciones. }\end{array}$ \\
\hline \multirow[t]{5}{*}{$\begin{array}{l}\text { Segmento } \\
\text { Académico }\end{array}$} & \multirow[t]{5}{*}{5 entrevistas } & $\begin{array}{l}\text { 11. Profesor investigador de la Universidad de Guadalajara, } \\
\text { Consultor en Mercadotecnia Política, Jefe de Departamento y } \\
\text { Miembro del Sistema Nacional de Investigadores CONACYT. }\end{array}$ \\
\hline & & $\begin{array}{l}\text { 12. Presidente del Consejo Técnico de Laboratorio de Vivienda y } \\
\text { Sustentabilidad CONACYT. }\end{array}$ \\
\hline & & $\begin{array}{l}\text { 13. Fundador del Centro de Estudios de Opinión de la Universidad } \\
\text { de Guadalajara, Director de División y miembros del Sistema } \\
\text { Nacional de Investigadores CONACYT. }\end{array}$ \\
\hline & & $\begin{array}{l}\text { 14. Fundador y Director de la Unidad de Inteligencia competitiva } \\
\text { del Instituto Tecnológico y de Estudios Superiores de } \\
\text { Occidente, Analista de Mercados. }\end{array}$ \\
\hline & & $\begin{array}{l}\text { 15. Miembro del Comité Técnico del Premio Gobierno y Gestión } \\
\text { Local del CIDE. Jefe de Departamento y Miembro del Sistema } \\
\text { Nacional de Investigadores CONACYT. }\end{array}$ \\
\hline $\begin{array}{l}\text { Segmento } \\
\text { Oferentes }\end{array}$ & 3 entrevistas & $\begin{array}{l}\text { 16. Director General de la empresa Polymétrix. Ramo: Realización } \\
\text { de estudios de opinión pública. Ex Director de la Unidad de } \\
\text { Muestreo del Centro de Estudios de Opinión de la Universidad } \\
\text { de Guadalajara. }\end{array}$ \\
\hline
\end{tabular}




\begin{tabular}{|l|l|l|}
\hline \multirow{2}{*}{} & $\begin{array}{l}\text { 17. Jefe de la Unidad de Encuestas del Centro de Estudios } \\
\text { Estratégicos para el Desarrollo de la Universidad de } \\
\text { Guadalajara. }\end{array}$ \\
\cline { 2 - 3 } & $\begin{array}{l}\text { 18. Consultor independiente de Estudios de Mercado y Branding y } \\
\text { ex colaborador de agencia de estudios de mercado líder en el } \\
\text { ramo. }\end{array}$ \\
\hline
\end{tabular}

Fuente: elaboración propia con base a la metodología de la investigación.

\section{DISCUSIóN}

\subsection{Los objetivos de estudio y su relación con las diferentes etapas del proceso electoral}

En el caso particular del segmento del mercado denominado electoral, se acentúa la demanda del recurso informativo derivado de los estudios de opinión en el periodo de tiempo en el que se llevan a cabo las campañas electorales y la fecha en la que se realizan los comicios electorales. Los entrevistados afirman que al inicio de la campaña el objeto de uso de los estudios de opinión comprende la posibilidad de identificar aquellas personalidades públicas que gozan de mayor conocimiento y aceptación por parte de la población, y quienes podrían desempeñarse competitivamente en una contienda. Afirman que los estudios de opinión pública representan un recurso útil para ellos, se presenta en el punto de partida de las campañas electorales con la finalidad de consultar a la ciudadanía respecto a las problemáticas que acontecen en la localidad, para elaborar propuestas de trabajo en respuesta a las demandas ciudadanas. Durante la campaña electoral, el equipo de campaña y/o colaboradores hacen uso de este instrumento de medición para conocer y monitorear el posicionamiento del (los) candidato(s) a través del tiempo que dura la campaña, para conocer las preferencias electorales de la ciudadanía y la intención de voto, entre otros temas de interés, culminando el día de la elección. Momento en que se llevan a cabo dos estudios con objetivos precisos y previamente definidos:

Encuesta de salida: Aplicadas a los votantes al salir de la casilla electoral después de haber realizado el voto, o bien, simulación de voto (sin validez oficial) al salir de la casilla a través de una urna y boleta invitando a los votantes que egresan de la casilla a repetir su voto simulado. Esta información es recopilada y enviada a una base receptora que integra la información para ser procesada con la finalidad de visualizar aproximaciones a los avances en los resultados de la jornada electoral.

Conteo rápido: Al terminar la jornada electoral se publica afuera de cada casilla el resultado del conteo de los votos que se efectuaron en dicha sección durante la jornada; esta información es recopilada de una muestra de casillas con la finalidad de realizar una estimación de los resultados electorales de manera paralela pero anticipada a los resultados oficiales que posteriormente dará a conocer el organismo oficial encargado de llevar a cabo las elecciones. 
Por otra parte, y una vez que las elecciones han culminado, aunado a herramientas cualitativas se llevan a cabo estudios que permiten identificar el razonamiento del voto y la animadversión. Una vez que el candidato electo inicia sus funciones, es pertinente generar información mediante las mencionadas metodologías para conocer las expectativas de la ciudadanía respecto a los gobiernos entrantes.

La figura 2 representa a través de una línea de tiempo los momentos y motivos de uso de estudios de opinión durante el proceso electoral.

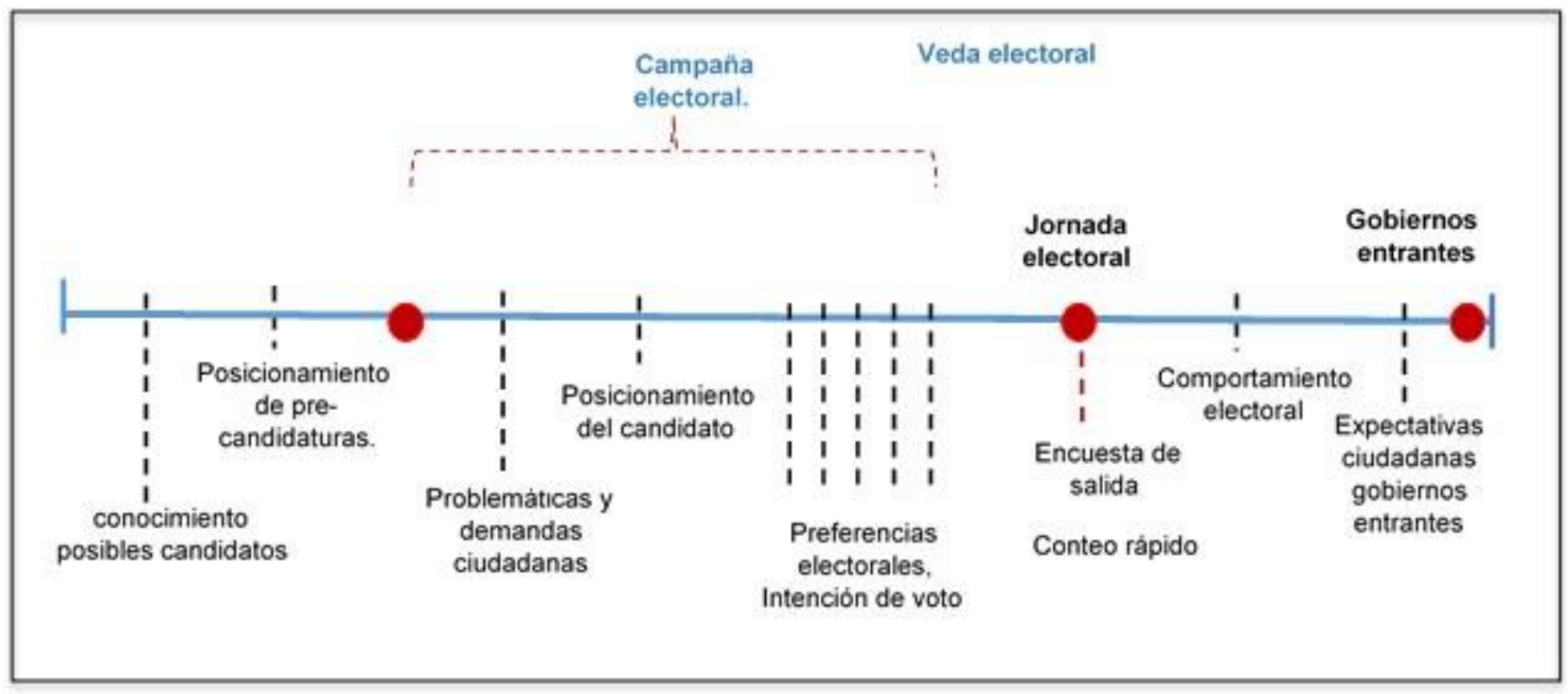

Figura 2: línea de tiempo y objetivos de estudio.

Fuente: elaboración propia.

El siguiente es un ejemplo de una encuesta electoral realizada por la empresa Consulta Mitosfky que con el objetivo de conocer la opinión ciudadana sobre la política y la sociedad en México realiza una encuesta a nivel nacional, misma que da a conocer en su portal de internet con información concerniente a las preferencias electorales previo a los comicios a celebrarse en el presente año. El marco muestral comprende el listado de secciones electorales en el país en los resultados en la elección federal en 2015, cuyo tamaño de muestra es de 1000 ciudadanos mayores de 18 años con credencial para votar distribuidos en todo el país, le encuesta se aplicó en el domicilio de los entrevistados mediante un cuestionario del 16 al 18 de marzo de 2018, aunque los resultados además muestran información comparativa a encuestas pasadas realizadas por esta misma agencia.

Los resultados de la encuesta presentan como primer lugar en las preferencias al candidato Andrés Manuel López Obrador quien representa a la alianza del Partido del Trabajo, Morena y Encuentro Social, en octubre de 2017 contaba con el 23,1\% de las preferencias y en la última medición realizada en marzo del 2018 obtiene el $29,5 \%$. 
Zarzosa Codocedo, A., Cota-Yáñez, R. y Rodríguez Cota, U. Importancia de los estudios de opinión pública: su aplicación y segmentación del mercado en México

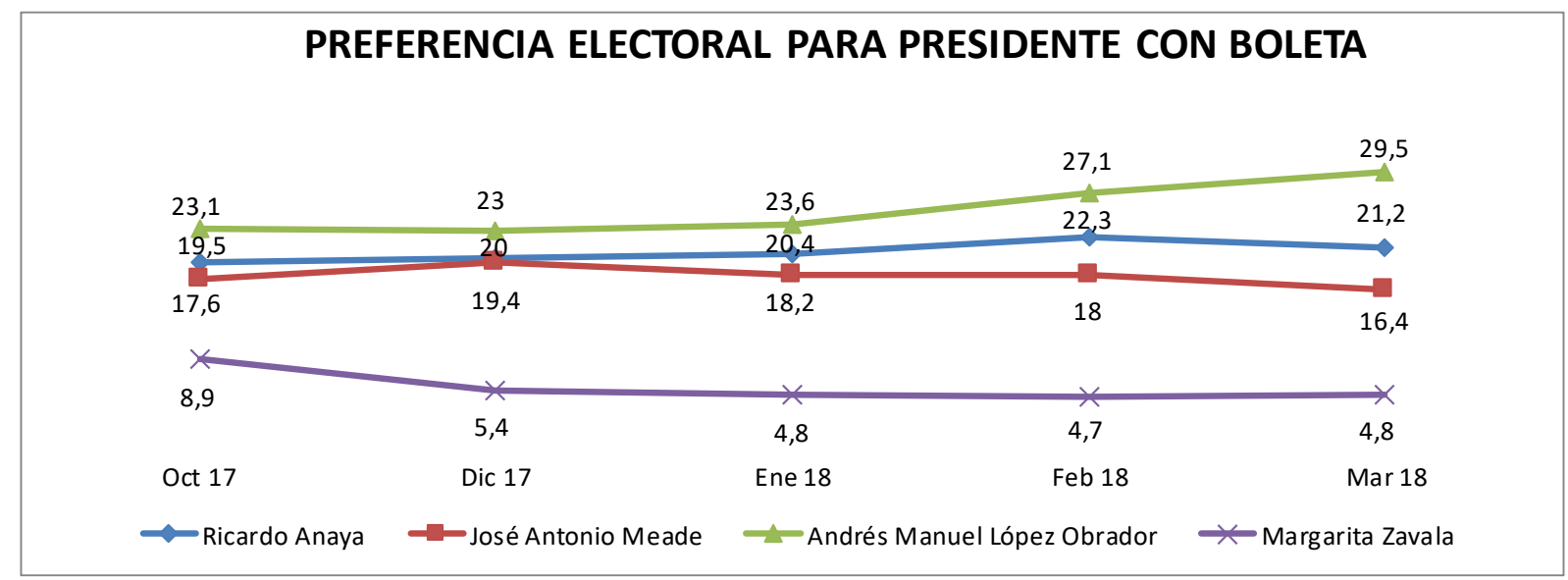

Gráfico 1: preferencia electoral para Presidente Oct 17-Marzo18 Consulta Mitofsky.

Fuente: consulta Mitofsky http://consulta.mx/

El siguiente cuadro muestra que el segundo lugar lo ocupa el Candidato Ricardo Anaya quien representa a la alianza del Partido Acción Nacional, Partido de la Revolución Democrática y Movimiento Ciudadano, en un comparativo de 4 encuestas llevadas a cabo entre diciembre de 2017 a marzo de 2018 en donde su avance global en dicho periodo es de 2,2\%, en tanto que el tercer lugar lo ocupa el candidato José Antonio Meade quien representa al Partido Revolucionario Institucional, Partido Verde Ecologista y Nueva Alianza, quien en relación con los resultados de diciembre 2017 a marzo de 2018 retrocede $3 \%$

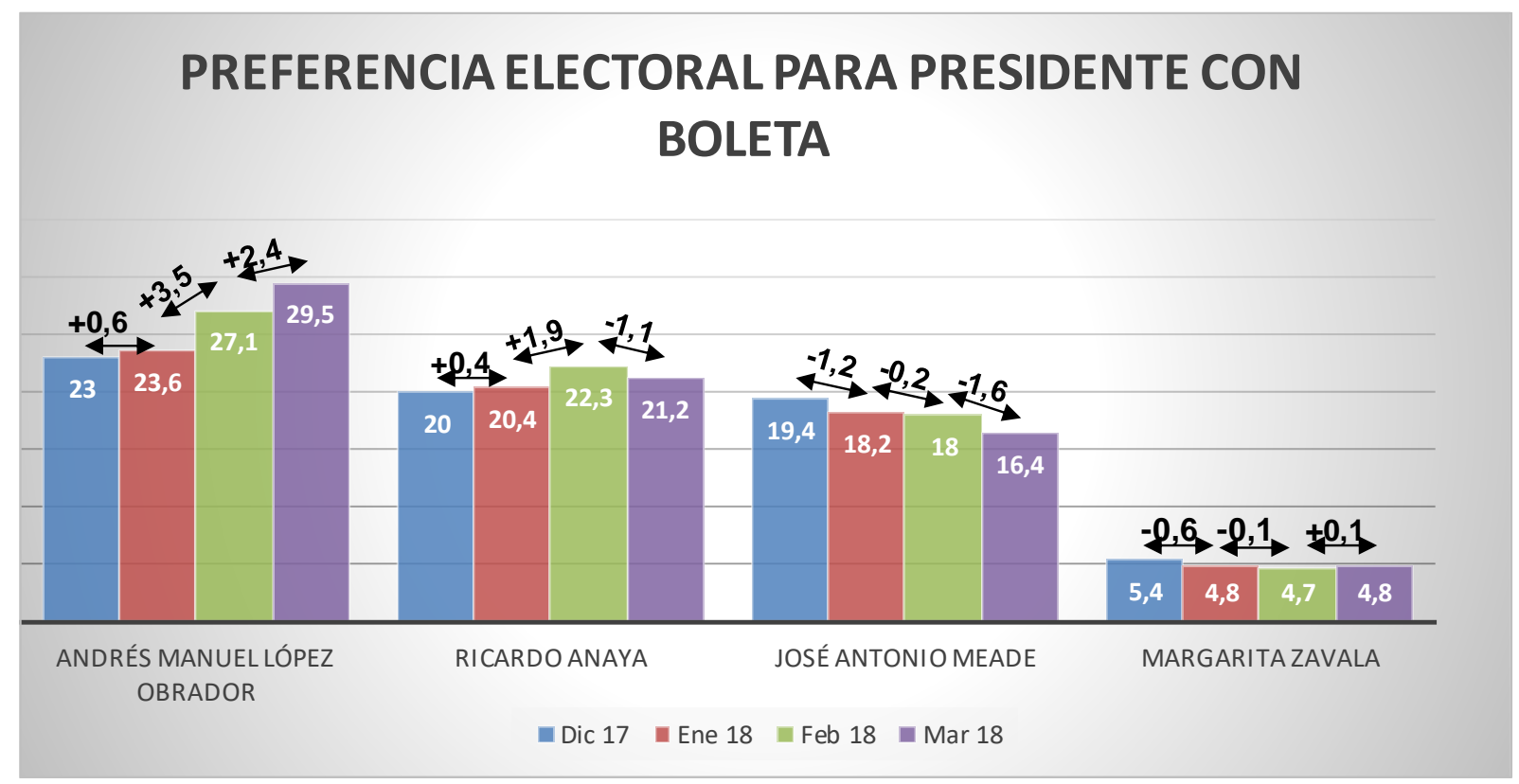

Gráfico 2: preferencia electoral para Presidente dic 17-Marzo18. Consulta Mitofsky. Fuente: consulta Mitofsky http://consulta.mx/

Cabe hacer mención que estas encuestas se han aplicado en el periodo previo a la campaña electoral y la más reciente corresponde a marzo de 2018, misma que da 
cuenta de la posición con la que inician los candidatos la campaña electoral oficial. Lo que demuestra que cuentan con un posicionamiento considerable como personajes públicos en el punto de partida.

Este estudio es una muestra del uso y aplicación que tienen los estudios de opinión pública en el ámbito electoral. Son herramientas que representan una brújula para los candidatos y sus estrategas de campaña, pero al mismo tiempo permite a la población enterarse del posicionamiento de sus candidatos, los avances y movimientos que se producen a través del tiempo en el desarrollo de la campaña electoral.

\subsection{Los estudios de opinión y la toma de decisiones}

Los entrevistados afirman que los estudios de opinión son una herramienta muy útil para tomar decisiones estratégicas, que permiten identificar el posicionamiento del candidato al cual apoyan, así como conocer sus fortalezas y debilidades en función a la opinión ciudadana, conocer la intención de voto de los electores, el margen de votantes indecisos entre otros. Identificar los puntos geográficos en donde se deben reforzar las acciones de campaña y los problemas a resolver a corto plazo. Hay quien afirma que un $80 \%$ de las decisiones que se toman durante la campaña se llevan a cabo tomando como insumo los estudios de opinión (ver figura 3).

- Identificar los personajes que cuentan con el reconocimiento y aceptación social.

- Medir el nivel de conocimientos de personajes (pre-candidaturas)

- Información acerca de las problemáticas ciudadanas, relacionadas con los servicios y politicas públicas: servicios seguridad.

- Evaluación de gobierno y servicios.

- Expediente con resultados de mediciones del candidato $=$ banco de información .

- Nivel de conocimiento y posicionamiento del candidato.(positivos y negativos)

- Participación ciudadana en procesos electorales anteriores.

- Información segmentada por sectores, socio-económicos, por género, respecto a preferencias electorales.

- Intención de voto por candidato, partido y fórmula ( posibles combinaciones).

- Animadversión ( rechazo por determinado personaje /partido).

Insumo que coadyuva en la decisión interna del partido para la elección del candidato. Mediciones con cierta periodicidad que permiten pedir el ritmo de avance. Tomar decisiones de estrategia durante el proceso electoral. Trazar rutas geográficas de trabajo en campaña

- Voto duro (definitivamente no cambiarian su voto).

- Voto nulo

- Información respecto a los electores

Figura 3: estudios de opinión y la toma de decisiones.

Fuente: Elaboración propia con base a la metodología de la investigación. 
La siguiente gráfica es un ejemplo que muestra con mayor detalle las particularidades de la intención de voto para Presidente al momento de iniciar la campaña electoral, en donde se puede identificar como voto volátil aquel de quien manifiesta cierta intención de voto cuya decisión puede cambiar de un momento a otro, el voto duro corresponde a aquellas personas quienes al ser encuestadas manifiestan que su intención de voto será inamovible. En este sentido quien cuenta con un mayor porcentaje de voto duro es el candidato Andrés Manuel López Obrador con el $19,2 \%$.

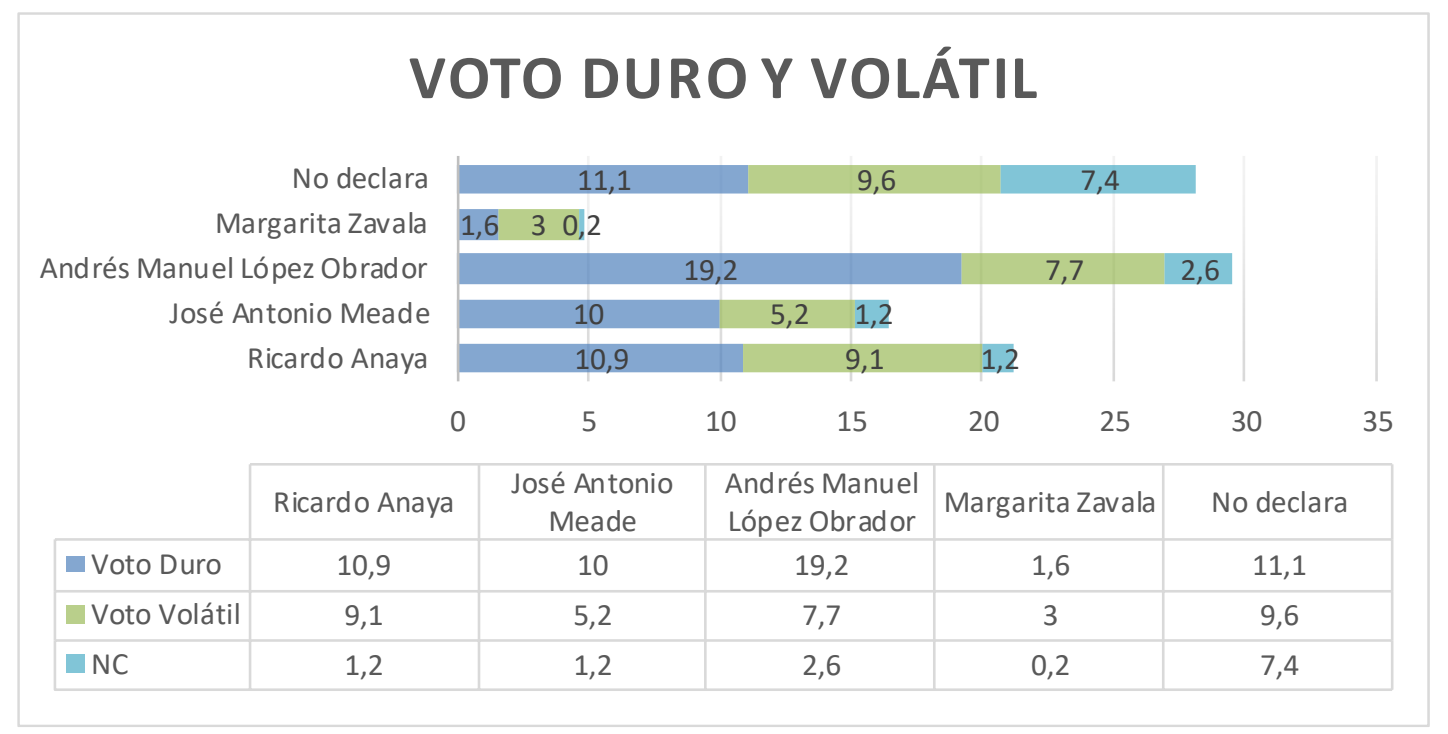

Gráfico 3: preferencia electoral para Presidente- voto duro y volátil Marzo18. Consulta Mitofsky.

Fuente: Consulta Mitofsky http://consulta.mx/

En lo referente al segmento de mercado correspondiente al sector público, los entrevistados comentan que los estudios en cuestión permiten retroalimentar a los servidores públicos respecto a la calidad y eficiencia con la que el servicio se ofrece a la ciudadanía, considerando que la información generada por este tipo de estudios se sustenta en la experiencia de uso y/o recepción del servicio público de parte del usuario/receptor del servicio, tanto en el ámbito de la educación, como en la salud, transporte, servicios públicos (agua, luz, drenaje).

De este modo, los estudios de opinión generan información que refleja el nivel de eficiencia del servicio en cuestión y el nivel de acercamiento a los parámetros establecidos por el mismo organismo, lo que en algunas ocasiones constituye una evidencia para acreditar el cumplimiento de indicadores de calidad establecidos por diferentes organismos externos acreditadores y certificadores en este ámbito.

Por citar un ejemplo, recientemente el Sistema del Tren Eléctrico Urbano (SITEUR) incorporó como parte de su servicio, la posibilidad de que los usuarios estacionen sus bicicletas en las diferentes estaciones; ofreciendo la posibilidad a los ciclistas de realizar un tipo de transporte mixto combinado entre la Línea del Tren y el transporte en bicicleta. La implementación de este programa se dio como resultado de un 
estudio de opinión que se llevó a cabo con la finalidad de recopilar las demandas de los usuario, así lo confirmó el titular de la dependencia, quien considera que los estudios de opinión son una herramienta que forma parte del plan de gobierno, ya que coadyuvan en la toma de decisiones, en particular en aquellas que tienen un impacto directo en la ciudadanía.

Los entrevistados mencionan como objetivos de estudio a resolverse con la realización de encuestas ciudadanas se encuentra el detectar las necesidades ciudadanas, identificar las necesidades de las ciudades en lo referente a obra pública, conocer la aceptación/rechazo en cuanto a la implementación de programas en específico.

Los siguientes son algunos de objetivos de estudio en este sector:

- Evaluación de servicios públicos.

- Evaluación de gobierno.

- Percepción y postura ciudadana sobre temas de controversia, polémica y coyuntura.

- Entre otros.

Los entrevistados en ese segmento afirman que no se pueden tomar decisiones a razón de una percepción personal o un criterio individual, y ningún directivo de la administración pública debe tomar decisiones al margen de la opinión ciudadana; de forma tal que los estudios de opinión son la herramienta ideal.

El siguiente es un ejemplo de un estudio correspondiente a este segmento del mercado -sector público- realizado con el objetivo de evaluar al gobierno en funciones del Estado de Jalisco en 2017. El estudio fue realizado por la empresa Consulta Mitofsky con fecha del 24 al 26 de marzo de 2017 a una muestra de 400 ciudadanos mayores de 18 años residentes en el Estado de Jalisco, aplicada cara a cara en su domicilio.

Los resultados del estudio muestran que poco menos de la mitad de la población $(44,4 \%)$ aprueba el gobierno estatal actual, lo que es menor en comparación con los resultados del mismo estudio realizados anteriormente en Junio de 2013 en donde el Gobernador contaba con el 54,3\% de aprobación.

En contraparte el porcentaje de la población que está de acuerdo con el Gobierno Estatal encabezado por Aristóteles Sandoval, presenta un aumento considerable de 14,8 puntos porcentuales en marzo de 2017 (44,4\% aprobación) en comparación con los resultados obtenidos en la primera encuesta de evaluación de Gobierno realizada en junio de 2013 en la que obtuvo 54,3\% de aprobación, misma que fue disminuyendo paulatinamente conforme se presentan las subsecuentes evaluaciones, obteniendo el porcentaje menor de aprobación en febrero de 2016 con el 39,9\%.

Los resultados demostrados en esta encuesta se comparan con la información generada por la misma agencia (Consulta Mitofsky) (gráfico 4), en donde se puede observar que el promedio de aprobación nacional de los gobernadores muestra la 
misma tendencia en cuanto a una disminución paulatina conforme al avance del tiempo de gobierno, detectado en las mediciones de esta encuesta realizada en 5 diferentes momentos: junio 2013, junio 2014, abril 2015, febrero 2016, y marzo 2017; dicha disminución comprende el punto de partida con una evaluación promedio de acuerdo del $62,1 \%$ a convertirse en 32,5\% de aprobación en marzo de 2017. En este sentido la evaluación del Gobierno del Estado de Jalisco en comparación con la evaluación de Gobierno Media en el país, se encuentra en una posición por arriba de la media más baja en la encuesta realizada en marzo de 2017 en la que obtiene $44,4 \%$ aprobación, frente a la media que obtiene en esta misma fecha el $32,5 \%$.

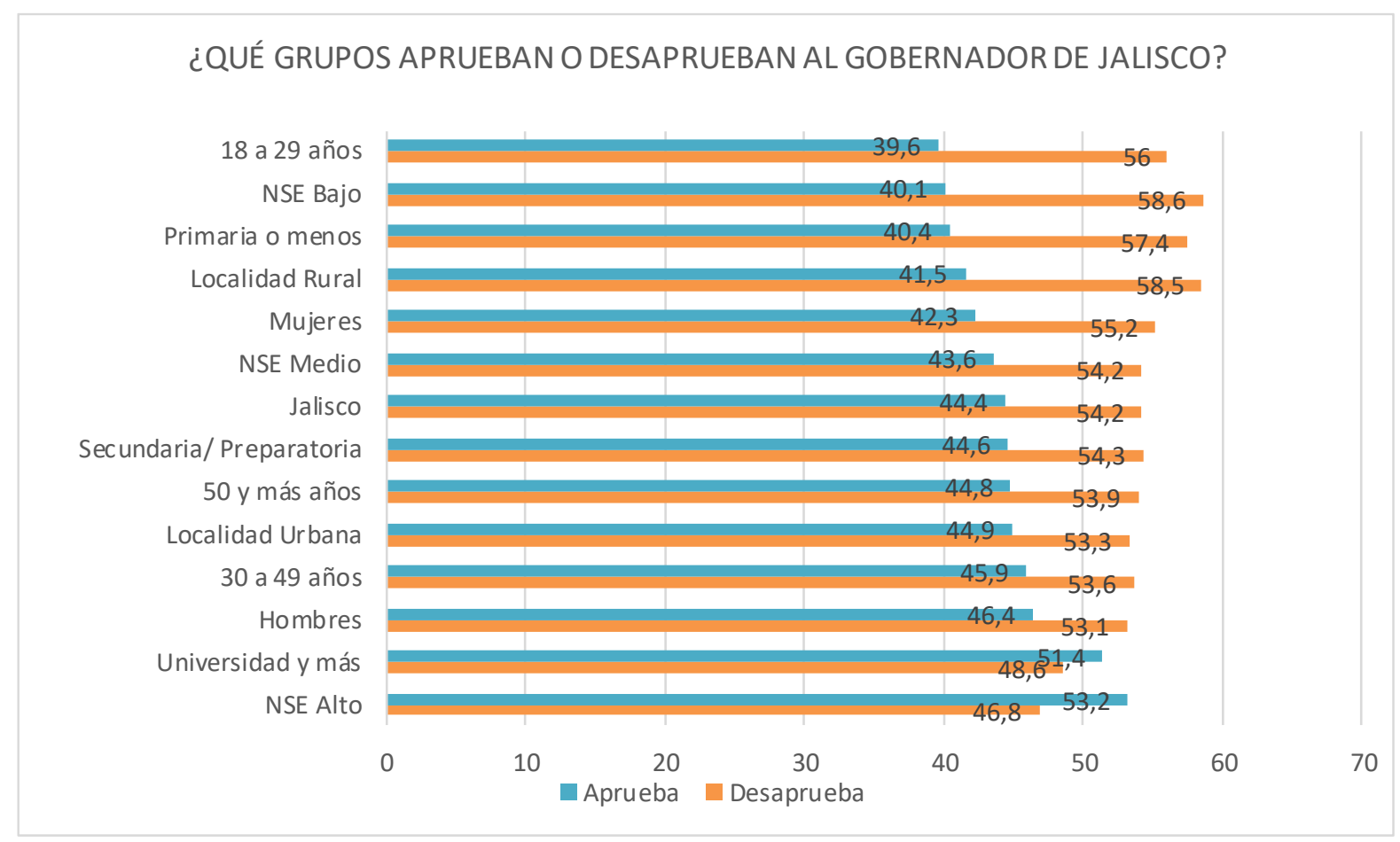

Gráfico 4: Jalisco evaluando a su Gobierno- Evaluación por grupos.

Fuente: Consulta Mitofsky http://consulta.mx/

En relación con los diferentes segmentos que conforman la población bajo estudio y en esta misma evaluación se puede identificar que el Gobierno en funciones obtiene una mejor evaluación en los grupos cuyo nivel socioeconómico es Alto, quienes cuentan con estudios universitarios, así como también en localidades urbanas. Mientras que el mayor porcentaje de desaprobación se encuentra entre los jóvenes de 18 a 29 años, en NSE Bajo y en localidades rurales.

\subsection{Los estudios de mercado en el sector privado}

Los entrevistados que representan al sector empresarial manifiestan llevar a cabo estudios de mercado integrados por diferentes instrumentos que en su conjunto permiten generar información que da respuesta a las necesidades particulares su organización, de tal manera que los estudios son tantos y tan variados como la gran 
Zarzosa Codocedo, A., Cota-Yáñez, R. y Rodríguez Cota, U. Importancia de los estudios de opinión pública: su aplicación y segmentación del mercado en México

diversidad de giros y ramos que conforman este sector, en donde se realizan estudios "ad hoc", a las particularidades de la empresa y sus circunstancias.

Las herramientas que integran el diseño de estos estudios son: encuestas, entrevistas de profundidad, grupos de enfoque, observación, técnicas proyectivas, estudios etnográficos.

Entre los entrevistados se identificaron diferencias en cuanto a la accesibilidad y realización de los estudios de cada empresa; ya que se presentan casos en los que la empresa tiene como parte de su estructura organizacional, un área específica destinada a la realización de sus propios estudios de mercado y por lo tanto se puede decir que son autogestivos en la generación de información; y por otra parte se presentan casos en los que la organización tiene un área de especialización en la identificación de necesidades de información, dedicada y especializada en la búsqueda de proveedores externos (agencias de investigación), para dar respuesta a las necesidades de información planteadas por la organización. Las dos anteriores en combinación también comprenden una realidad en este sector, en donde solo una parte de los estudios de mercado se realiza "in house" y la otra parte a través de la compra a proveedores.

En ambos casos las decisiones que giran en torno al producto y/o servicio son de gran escala, y repercuten directamente en las ventas, por lo que los directivos asignan el merecido valor a la información que les permite tomar decisiones con fundamentos precisos y en donde la realización de este tipo de estudios forma parte de la cotidianeidad en el funcionamiento de la organización y representa un elemento fundamental para el éxito.

En este mismo segmento se identifica una fracción del mercado conformado por aquellas empresas que no cuentan con la proveeduría de información interna y recurren a la realización de estudios de mercado a través de agencias especializadas al exterior de la empresa.

Existe una gran diversidad de objetivos de estudio que se realizan en este segmento, entre los que destacan: Nivel de conocimiento de la marca, Participación de mercado, Evaluación del producto/servicio, Percepción e imagen del producto, Comportamiento de la participación de mercado: mediciones periódicas que permiten identificar el movimiento del mercado entre las diferentes marcas, Información de la competencia, Previo al lanzamiento de un producto: aceptación de cualidades y características, Identificación de nicho de mercado, Identificación de causales de baja en las ventas.

Para este grupo de entrevistados, los estudios de mercado constituyen además, una evidencia para responder a indicadores claves de desempeño que requieren de una demostración de fuentes primarias respecto a la posición del producto en el mercado, sus fortalezas, su posición frente a la competencia, entre otros.

Los entrevistados requieren de la generación de información a través de la realización de estudios de mercado para dar respuesta a interrogantes tales como 
por ejemplo el tener la oportunidad de identificar las razones que causan una baja en las ventas, identificar las marcas y productos competidores que el consumidor conoce, identificar también a quien le compra ya sea el consumidor o el cliente de la marca/producto en particular, identificar fidelidades anteriores a otras marcas y las causales de la fractura de relación de compra, conocer la evaluación del servicio por parte del usuario, identificar las marcas líderes en el mercado según el Top of Mind, conocer las acciones que realiza la competencia entre las que se encuentra la revisión y análisis de sus campañas, sus precios, entre otras muchas.

El siguiente es un fragmento de un estudio de mercado realizado por la empresa mercawise, denominado Estudio de Mercado sobre uso de gimnasios y complementos alimenticios, aplicado a 209 usuarios de su página web, seleccionados a través del método aleatorio simple.

El estudio de mercado muestra que el $20,6 \%$ de los entrevistados que acostumbran hacer ejercicio en el gimnasio han estado suscritos a este en un periodo de tiempo de 6 a 12 meses, mientras que el 19,1\% de 3 a 6 meses y el $18,7 \%$ de 1 a 3 meses (tabla 2 ).

Tabla 2. Estudio de mercado Uso de gimnasios y complementos alimenticios. Tiempo gimnasio.

\begin{tabular}{|lcr|}
\hline \multicolumn{3}{|c|}{ ¿Cuánto tiempo has estado inscrito a un gimnasio? } \\
\hline - Menos de un mes & 13 & $6,2 \%$ \\
\hline - De 1 a 3 meses & 39 & $18,7 \%$ \\
\hline - De 3 a 6 meses & 40 & $19,1 \%$ \\
\hline - De 6 a 12 meses & 43 & $20,6 \%$ \\
\hline - De 1 año a 2 años & 21 & $10 \%$ \\
\hline - De 2 años a 3 años & 12 & $5,7 \%$ \\
\hline - Más de 3 años & 11 & $5,3 \%$ \\
\hline - Nunca & 20 & $14,4 \%$ \\
\hline
\end{tabular}

Fuente: https://www.mercawise.com/estudios-de-mercado-en-mexico/estudio-demercado-sobre-el-uso-de-gimnasios-y-complementos-vitaminicos

La tercera parte de la población encuestada $(36,4 \%)$ asiste al gimnasio para hacer ejercicio de 2 a 3 veces por semana y una cuarta parte de los entrevistados $(26,8 \%)$ asiste de 4 a 6 veces, mientras que solo el 9,6\% asiste a ejercitarse a este tipo de lugares todos los días. En donde la razón más importante para escoger el gimnasio de la preferencia de los usuarios, radica en que las instalaciones deben ser de su agrado $(20,1 \%)$ y el precio acorde a sus posibilidades según lo afirma el $18,7 \%$ de los encuestados así como también el hecho de que el gimnasio ofrezca atención personalizada a sus usuarios $17,7 \%$.

El motivador principal para acudir al gimnasio para hacer ejercicio es la salud, según el $41,1 \%$ seguido del interés por mejorar su aspecto físico para el $33 \%$ y 
mejorar la condición física para el 18,2\%. El periodo de pago de preferencia del $71,3 \%$ es en mensualidades, y solo un $10 \%$ manifiesta disponibilidad de pago bimestral y el $8,6 \%$ de forma trimestral.

Respecto a los suplementos alimenticios y el nivel de conocimiento de los encuestados, más de la mitad $(63,2 \%)$ manifiesta tener conocimiento sobre estos, sin embargo este conocimiento es limitado al referirse a este como "tengo una idea, pero no los conozco del todo", mientras que una cuarta parte $(25,8 \%)$ de la población manifiesta tener conocimiento y e1 $11 \%$ no conoce.

El mercado potencial de compra de complementos alimenticios como parte del régimen de alimentación en combinación con ejercicios como parte de los servicios del gimnasio es viable para el $26,8 \%$ de los entrevistados mientras que el costo de estos sea razonable; sin embargo el $37,3 \%$ considera muy importante el consumo de este tipo de suplementos para ver resultados del ejercicio por lo que sí estarían definitivamente dispuestos a tomarlos con un costo extra. Por otra parte el $29,2 \%$ se encuentra se encuentra indeciso al respecto y solo el 6,7\% se muestra totalmente renuente a consumir los productos mencionados.

\subsection{Las encuestas en la investigación académica}

La investigación que se lleva a cabo en las universidades a manos de los expertos y especialistas en diversos temas, persigue un beneficio para la sociedad, y por lo tanto los resultados de este tipo de investigación se aplica a los diferentes ámbitos. Así es como la investigación aplicada requiere de la interacción con los actores sociales, y la recopilación de información de ciertos grupos, para lo cual, entre otros mecanismos, la encuesta es una herramienta que permite al investigador obtener esta información.

Los entrevistados en este segmento integrado por académicos quienes como parte de sus funciones realizan investigación, refieren como estudios que requieren de la información generada a través de encuestas, los siguientes: Demografía, Estudios de género, Calidad de vida, Dinámica demográfica, migratoria, Vivienda, Consumo energético, Seguridad en la vivienda, Segregación espacial, Ciudad, urbanismo y territorio, Percepción de espacios públicos y áreas verdes, Uso de agua y energía, Políticas y programas de vivienda y planeación, Educación, Entre otros.

Cabe destacar la relevancia que tiene en este sector la información secundaria, ya que aporta elementos de gran valía para investigaciones de largo aliento. En este segmento se identifica a la aplicación de este tipo de estudios en dos sentidos; en el primero se encuentra la información secundaria en donde los estudios son realizados por organismos externos y la información generada representa un insumo valioso que sustenta la investigación que el profesor realiza, y por otra parte se encuentra la encuesta que se lleva a cabo como parte de la investigación, misma que genera información detallada y responde a objetivos particulares a la investigación. 
Conforme a la opinión de los expertos en esta materia entrevistados; las encuestas abonan al desarrollo del conocimiento en particular a las ciencias sociales, en el caso de la economía y en las disciplinas administrativas ya sea en el ámbito comercial o de investigación; para ambas las encuestas representan un insumo de considerable importancia. Desde su perspectiva el $50 \%$ de las problemáticas que acontecen pueden ser abordadas a través de este tipo de estudios, aunado a que existen importantes instituciones como el INEGI, organismo que a juicio de los entrevistados se ha desarrollado favorablemente en los últimos 15 años, y genera un acervo de información valioso y a tomar en consideración como fuente de información secundaria.

Los académicos entrevistados consideran que la encuesta como herramienta ofrece respuestas a las preguntas o temas muy puntales y específicos que no se han abordado con anterioridad como producto de otra fuente. Mencionan organismos generadores de información a través de encuestas de tipo institucional tal es el caso de la información que produce el INEGI entre las encuestas que mencionaron se encuentran: Censo de población y vivienda, censo económico, encuesta industrial mensual (EIM), encuesta anual de comercio (EAC), encuesta nacional de empleo, salarios, tecnología y capacitación en el sector manufacturero (ENESTyC), entre otras. Esta información, entre otras, está a disposición del público de manera abierta, sin embargo, comentan que la forma en la que el organismo ofrece la información requiere de ciertas habilidades y/o conocimiento sobre la utilización y el manejo de bases de datos.

El siguiente es un ejemplo de una encuesta que realiza el Instituto Nacional de Estadística y Geografía (INEGI) sobre la disponibilidad de Tecnologías de la Información y Comunicación (TIC) en los hogares y de su uso por los individuos, denominada (ENDUTIH) Encuesta Nacional sobre Disponibilidad y Uso de las Tecnologías de la Información en los Hogares en donde, se da continuidad a la encuesta iniciada en el año 2015, aplicada ahora en 2017 en 32 entidades federativas y en 49 ciudades apoyado por la Secretaria de Comunicaciones y Transportes y por el Instituto Federal de Telecomunicaciones. La encuesta realizada se aplica a un miembro del hogar seleccionado de manera aleatoria quien proporciona información sobre el uso de tecnologías de la información mediante la aplicación de un cuestionario estructurado.

La ENDUTIH 2017 revela que 17,4 millones de hogares disponen de Internet ( $50,9 \%$ del total nacional), ya sea mediante una conexión fija o de tipo móvil, lo que significa un incremento de 3,9 puntos porcentuales respecto del año anterior.

La encuesta presenta resultados para los ámbitos urbano y rural; en este sentido, la encuesta muestra que 86 de cada 100 usuarios de Internet en el país se ubican en áreas urbanas, y el resto (14 de cada 100) se encuentran en áreas rurales.

Al revisar comparativamente la encuesta 2017 frente a la encuesta 2016 se puede observar que el principal motivo para el uso de internet comprende la obtención de 
Zarzosa Codocedo, A., Cota-Yáñez, R. y Rodríguez Cota, U. Importancia de los estudios de opinión pública: su aplicación y segmentación del mercado en México

información, el entretenimiento y la comunicación con $96,9 \%, 90,0 \%$ y $91,4 \%$ respectivamente.

La encuesta permite tener mediciones para hacer comparativos con información de otros países, así se puede identificar la posición que ocupa México en el uso de internet frente al índice de otros países, en donde Suecia, Reino Unido, Corea del Sur, Japón y Alemania, nueve de cada diez personas son usuarios de Internet; mientras que en México la proporción es seis de cada diez personas.

Aunado a lo anterior la encuesta también presenta información acerca de la telefonía celular de manera global y por entidad federativa, por tipo de equipo y por tipo de conexión, así como también indaga acerca de la instalación de aplicaciones, además de uso de equipo de cómputo y acceso a televisión digital.

\section{CONCLUSIONES}

Los estudios de opinión pública y de mercado constituyen una importante industria nacional que produce miles de unidades anualmente, cuya oferta está integrada por gran diversidad de empresas localizadas en toda la extensión nacional cuya diversidad radica en la especialización del (los) tipo(s) de estudios que realizan, lo que está directamente relacionado con la tipificación de 4 tipos de estudios y desde el punto de vista mercadológico, esta variedad constituye una línea de 4 variables de productos entre las que se encuentran: Estudios de opinión pública, estudios electorales, investigaciones de mercado y asesoría y consultoría en mercadotecnia.

Desde la perspectiva de la demanda, se puede identificar una clara segmentación por tipo de producto que en ese caso lo definen los objetivos del estudio, de forma tal que la segmentación del mercado en cuestión está integrada por el segmento electoral, el segmento del sector público, el sector privado y la academia; todos ellos requieren, generan y demandan información procedente de determinada población.

En el caso del segmento electoral los objetivos del estudio están directamente ligados los tiempos en los que se llevan a cabo los procesos electorales, periodo en el que se llevan a cabo diversidad de estudios entre los que destacan: posicionamiento de precandidaturas y candidaturas, preferencias electorales e intención de voto, encuesta de salida y conteo rápido, entre otras.

En el segmento del sector público se realizan estudios que permiten retroalimentar a la función pública respecto a la eficiencia y calidad de los servicios públicos que brindan a la ciudadanía, a través de estudios de diversas temáticas, entre las que destacan: evaluación de servicios públicos, evaluación de gobierno, percepción y opinión ciudadana respecto a eventos coyunturales, entre otros.

En el sector privado los estudios integran una mayor complejidad como parte de su metodología, conformada por diversas técnicas cuyo diseño comprende la combinación de éstas en función a los objetivos de investigación. Entre las técnicas

Revista de Comunicación de la SEECI. 15 julio, 2020 / 15 noviembre, 2020, nº 52, 95-117 
que conforman dicha metodología se encuentran las encuestas, entrevistas de profundidad, grupos de enfoque, observación, técnicas proyectivas, estudios etnográficos, entre otros.

Este segmento, a su vez se subdivide en tres grupos, el primero está integrado por aquellas empresas que tienen capacidad auto-gestiva en la generación de información quienes como parte interna de su organización cuentan con un departamento que se dedica a la realización de estudios de mercado de principio a fin, acorde con los requerimientos internos, un segundo grupo de empresas que cuentan con un equipo de personas que están inmersas en las necesidades de información de la empresa que se especializan en la búsqueda y selección de proveedores en materia de investigación de mercados y contratan los servicios de investigación a proveedores externos y por último un tercer grupo integrado por aquellas empresas que no cuentan con equipo alguno para ocuparse en materia de investigación.

Entre los tipos de estudios que se realizan en este segmento se encuentran, estudio sobre nivel de conocimiento de marca, medición de participación de mercado, evaluación del producto/servicio, percepción/imagen del producto, entre otros.

La investigación descriptiva del mercado de los estudios de opinión pública y de mercado permite dimensionar el grado de especialización y profesionalización que la constituye como una industria proveedora de información de alto grado de confiabilidad tanto a nivel nacional como internacional, integrada por profesionistas que se especializan en la materia, variables todas que en su conjunto se traducen en una alta calidad de la información para la toma de decisiones.

\section{REFERENCIAS}

Asociación Mexicana de Agencias de Investigación de Mercado. (2014). Estudio Anual de la Industria de la Investigación de Mercados y Estudios de Opinión Pública en México. México: sin editorial.

Aaker, D. y Kumar, V. (2013). Investigación de Mercados. México: Editorial Limusa.

Berúmen y Asociados. Recuperado de http://www.berumen.com.mx/

Demotecnia. Recuperado de http://www.demotecnia.com.mx/dmt/

D'Adamo, O. et al. (2007). Medios de Comunicación y Opinión Pública. España: Mc Graw Hill.

Grupo KP. Extraído de http://www.kp.com.mx/

Consulta Mitosky. Recuperado de http://consulta.mx/ 
Zarzosa Codocedo, A., Cota-Yáñez, R. y Rodríguez Cota, U. Importancia de los estudios de opinión pública: su aplicación y segmentación del mercado en México

Investigaciones Sociales Aplicadas, ISA. Recuperado de http://www.isa.org.mx/

Lamb, C. W., Hair, J. F., \& McDaniel, C. (2012). Marketing 11e. México: CENGAGE, Learning.

Mercawise. Recuperado de https://www.mercawise.com/estudios-de-mercado-enmexico/estudio-de-mercado-sobre-el-uso-de-gimnasios-y-complementosvitaminicos/

Rivadeira, R. (1995). La Opinión Pública, Análisis, Estructuras y Metadatos para el estudio. México: Editorial Trillas.

Ruíz, J. (1997). Introducción a la Tradición Clásica de la Opinión Pública. Madrid: Tecnos.

Sharon, L. (2000). Muestreo: Diseño y Análisis. España: Thompson.

Young, K. (1995). La Opinión Pública y la Propaganda. España: Paidós.

\section{AUTORES:}

\section{Alejandra Zarzosa Codocedo}

Profesora Investigadora de la Universidad de Guadalajara. Maestra en Mercadotecnia Global por el Instituto Tecnológico y de Estudios Superiores de Occidente. Licenciada en Mercadotecnia, se ha especializado en levantamiento de Estudios de Opinión. Líneas de investigación: mercadotecnia, estudios de mercado, desarrollo local.

Alejandra.Zarzosa@redudg.udg.mx

\section{Rosario Cota-Yáñez}

Profesora-Investigadora del Departamento de Estudios Regionales de la Universidad de Guadalajara (DER/INESER). Licenciada en Economía. Maestra en Desarrollo Regional con especialidad en Políticas Públicas. Doctora en Ciencias Sociales con especialidad Desarrollo Regional. Pertenece al Cuerpo Académico Reestructuración Productiva y Estudios Locales UDG 643. Líneas de investigación: desarrollo local, pequeñas empresas, políticas públicas.

maria.cota@academicos.udg.mx

Orcid ID: https://orcid.org/0000-0002-1866-7191

Google Scholar:

https://scholar.google.com.mx/citations?user=f1yDbrAAAAAJ\&hl=es\&oi=sra

Researchgate: https://www.researchgate.net/profile/Maria Yanez22

Redalyc: http://www.redalyc.org/autor.oa?id=25377

\section{Ulises Rodríguez Cota}

Pasante de la licenciatura en Comunicación y Periodismo Deportivo. Instituto José Ramón Fernández. Actualmente difunde por redes sociales (Facebook e Instagram) 
Zarzosa Codocedo, A., Cota-Yáñez, R. y Rodríguez Cota, U. Importancia de los estudios de opinión pública: su aplicación y segmentación del mercado en México

información relevante sobre resultados de partidos de futbol soccer, americano, baseball entre otros.

ulises.101@hotmail.com

Orcid ID: https://orcid.org/0000-0003-3951-1695 